EPJ Web of Conferences 47, 02004 (2013)

DOI: $10.1051 /$ epjconf/20134702004

(C) Owned by the authors, published by EDP Sciences, 2013

\title{
Detecting circumbinary planets: A new quasi-periodic search algorithm
}

\author{
D. Armstronga and D. Pollacco
}

Department of Physics, University of Warwick, Gibbet Hill Road, Coventry CV4 7AL, UK

\begin{abstract}
We present a search method based around the grouping of data residuals, suitable for the detection of many quasi-periodic signals. Combined with an efficient and easily implemented method to predict the maximum transit timing variations of a transiting circumbinary exoplanet, we form a fast search algorithm for such planets. We here target the Kepler dataset in particular, where all the transiting examples of circumbinary planets have been found to date. The method is presented and demonstrated on two known systems in the Kepler data.
\end{abstract}

\section{INTRODUCTION}

Since the recent discovery of several circumbinary planets (e.g. [1-4]), the existence of these planets has sparked much work attempting to understand their formation and subsequent evolution. They represent a class of systems which form and exist under perturbations, both gravitational and thermal, from the host binary stars. As such, discovery of these systems is important for placing constraints on theory and models of how protoplanetary disks and planetessimals react to these conditions. In the case of transiting planets, discoveries are complicated by significant variations in transit timing, duration, and shape, as the planet transits what is in effect a "moving target".

In order to aid future discoveries we present progress on a search algorithm capable of detecting quasi-periodic signals, such as those presented by circumbinary planets. It should prove of use to current and future searches for these planets, as well as potentially many other quasi-periodic signals.

\section{ALGORITHM}

\subsection{Methodology}

Our search algorithm is based around the grouping of datapoint residuals. A series of trial periods are sampled (such as in for example the BLS algorithm [5]), and at each period the data is phase-folded and a filled-in box is "fit". Datapoints outside the box are iterated over forming a least-square sum, whereas data points within the box do not contribute to the sum. In this way departures from the mean of the dataset are "grouped", producing a periodogram spike where such departures are concentrated best together. The free parameters are the width and depth of the box - limiting the depth can be used to exclusively target low magnitude signals, and the width can be set by knowledge of the physics involved. An example for Kepler-16b [1] is shown in Fig. 1, where the box has been fit to the observed planetary transit signal.

\footnotetext{
ae-mail: d.j.armstrong@warwick.ac.uk
}

This is an Open Access article distributed under the terms of the Creative Commons Attribution License 2.0, which permits unrestricted use, distribution, and reproduction in any medium, provided the original work is properly cited. 


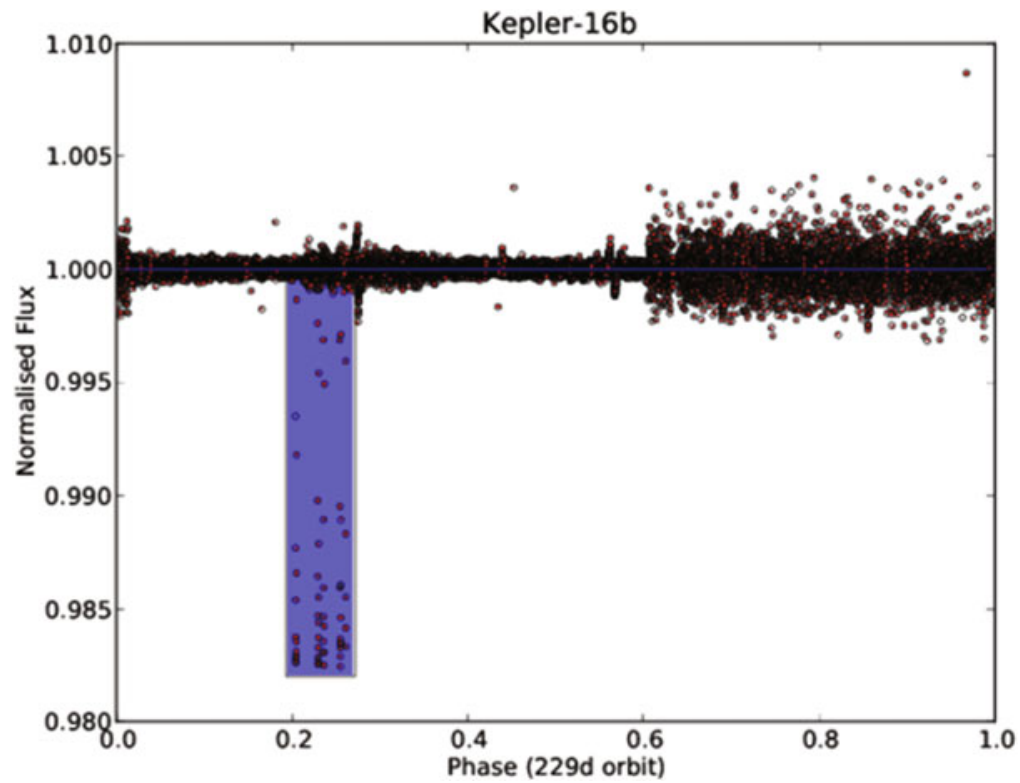

Figure 1. Kepler data of the circumbinary system Kepler-16, phase-folded at the planetary orbital period of [1]. A box is overlaid, showing a potential model fit to the data.

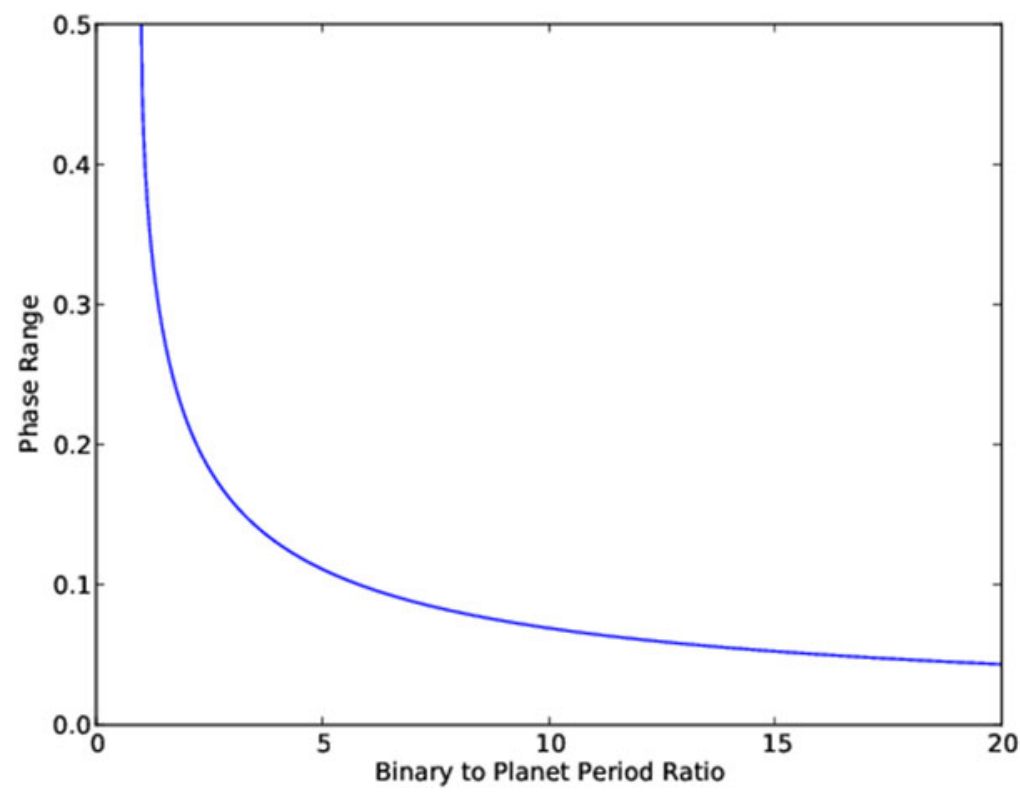

Figure 2. The dependence of the phase range - the width of the region in phase which potential transits of a circumbinary planet can occur in - on the planetary to binary period ratio.

\subsection{Limits on the transit timing variations of circumbinary planets}

It is possible to use the geometry of a binary system to place limits on the transit timing variations which a given circumbinary planet can exhibit. As the extent of the orbit of the host binary stars on the sky is limited, so too are the timing variations of the planet. A full explanation and derivation is available 


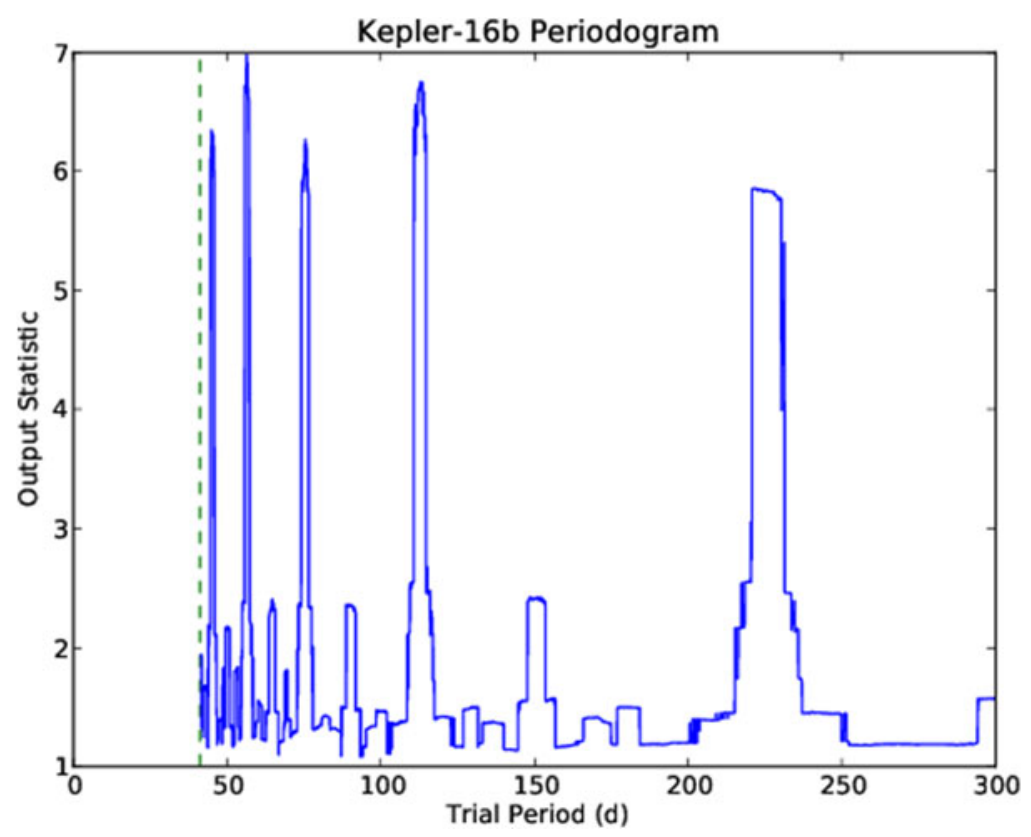

Figure 3. The periodogram obtained through running our search algorithm on the known circumbinary system Kepler-16. Each peak shows either the actual or a resonance thereof the planetary orbital period.

from Armstrong et al. (2013, submitted to MNRAS), where specific equations are presented. Figure 2 shows the general dependence of the phase range (the proportion of the planet's orbital period which it spends in a position where transits of either host star are possible) on the planet to binary period ratio. This maximum phase range can be used to place limits on potential transit timing variations, and set the width of the search box described in Section 2.1.

\section{DEMONSTRATION}

\subsection{Kepler-16b}

We first demonstrate the effectiveness of this search method on Kepler-16b, the first transiting circumbinary planet discovered. The transit signal of this planet is especially clear (transits of 1-2\% depth), and so it represents a good first test. Figure 3 shows the resultant periodogram we generate. There are several points to note. Firstly, the detection is clear: every peak in the periodogram represents a resonance of the planet's orbital period. This brings out a further point - this method is very weak to resonances. The actual planetary period is close to $229 \mathrm{~d}$ - each other peak in Fig. 3 is a resonance of this. As such, the purpose of this search algorithm is primarily detection rather than the pinpointing of an accurate orbital period. Finding specific periods in quasi-periodic systems is generally non-trivial, requiring in the case of circumbinary planets detailed modelling of the system.

\subsection{PH1}

PH1 $[6,7]$ is a more recent discovery. It shows much shallower transits than Kepler-16b, 0.15\%, and is such a much more difficult signal. Figure 4 shows the resultant periodogram. The detection is still clear, with each peak in the periodogram still representing a signal from the planet. 


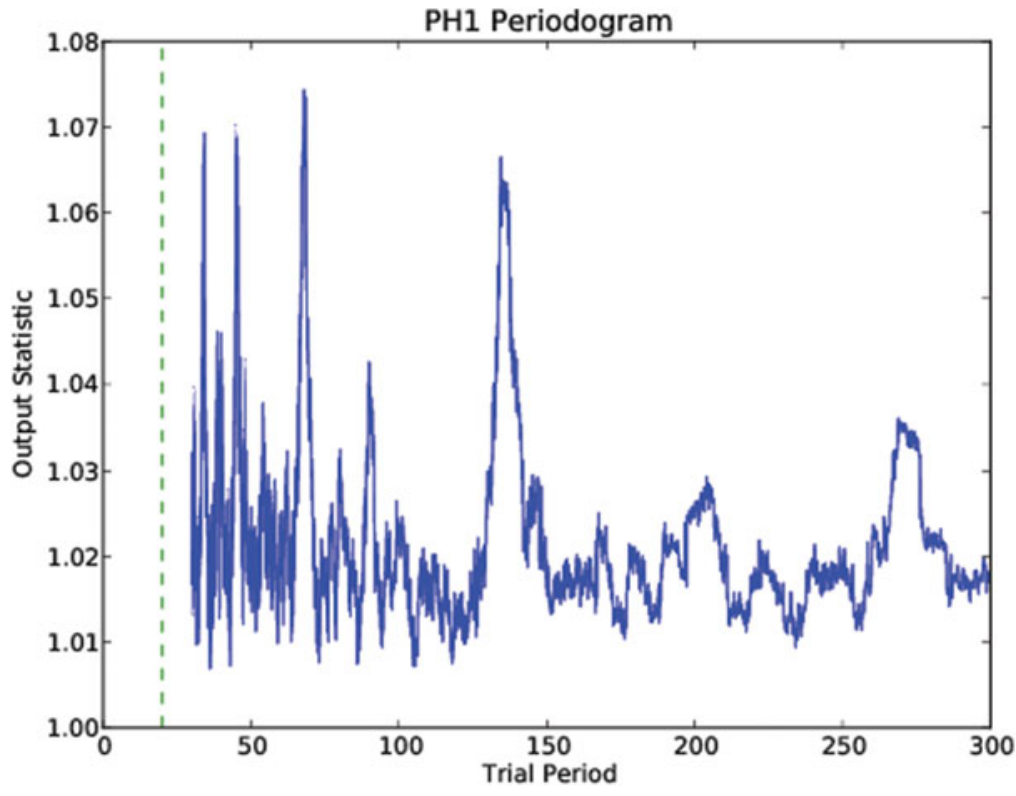

Figure 4. As Fig. 3 for the circumbinary planet PH1.

\section{PRELIMINARY RESULTS}

We have applied the above search method to the Kepler dataset. The full process involves various steps designed to remove stellar and instrumental variability, and will be described in full at a later date. At present, we have found 22 systems showing signals which indicate the presence of a third body. The majority of these will be either triple star systems or blends - systems where the transit-like signal arises from a fainter background star. Based on the quality of the signal obtained, we are following up 8 of these candidates in particular as signals more likely to be caused by the presence of a circumbinary planet. If even a few of these candidates prove to be true transiting planets, they will represent a significant increase in the number of such planets known. Based on this search and tests of its efficacy, we are working towards a quantified population study of planets in circumbinary orbits.

\section{References}

[1] L.R. Doyle, J.A. Carter, D.C. Fabrycky, R.W. Slawson, S.B. Howell, J.N. Winn, J.A. Orosz, A. Prsa, W.F. Welsh, S.N. Quinn et al., Science 333, 1602 (2011)

[2] W.F. Welsh, J.A. Orosz, J.A. Carter, D.C. Fabrycky, E.B. Ford, J.J. Lissauer, A. Prsa, S.N. Quinn, D. Ragozzine, D.R. Short et al., Nature 481, 475 (2012)

[3] J.A. Orosz, W.F. Welsh, J.A. Carter, E. Brugamyer, L.A. Buchhave, W.D. Cochran, M. Endl, E.B. Ford, P. MacQueen, D.R. Short et al., The Astrophysical Journal 758, 87 (2012)

[4] J.A. Orosz, W.F. Welsh, J.A. Carter, D.C. Fabrycky, W.D. Cochran, M. Endl, E.B. Ford, N. Haghighipour, P.J. MacQueen, T. Mazeh et al., Science 337, 1511 (2012)

[5] G. Kov cs, S. Zucker, T. Mazeh, Astronomy and Astrophysics 391, 369 (2002)

[6] M.E. Schwamb, J.A. Orosz, J.A. Carter, W.F. Welsh, D.A. Fischer, G. Torres, A.W. Howard, J.R. Crepp, W.C. Keel, C.J. Lintott et al., The Astrophysical Journal (2012)

[7] V.B. Kostov, P. McCullough, T. Hinse, Z. Tsvetanov, G. Hébrard, R. Díaz, M. Deleuil, J.A. Valenti, arXiv.org (2012) 